\title{
Thrombosis in the critically ill neonate: incidence, diagnosis, and management
}

\author{
Alex Veldman' \\ Marcel F Nold ${ }^{2}$ \\ Ina Michel-Behnke ${ }^{3}$ \\ 'Monash Newborn and Ritchie \\ Centre for Baby Health Research, \\ Monash Medical Centre and Monash \\ Institute of Medical Research, \\ Melbourne, VIC, Australia; ${ }^{2}$ Dept \\ of Medicine, University of Colorado \\ Health Sciences Center, Denver, CO, \\ USA; ${ }^{3}$ Dept of Pediatric Cardiology, \\ Pediatric University Hospital, Vienna, \\ Austria
}

\begin{abstract}
Among children, newborn infants are most vulnerable to development of thrombosis and serious thromboembolic complications. Amongst newborns, those neonates who are critically ill, both term and preterm, are at greatest risk for developing symptomatic thromboembolic disease. The most important risk factors are inflammation, DIC, impaired liver function, fluctuations in cardiac output, and congenital heart disease, as well as exogenous risk factors such as central venous or arterial catheters. In most clinically symptomatic infants, diagnosis is made by ultrasound, venography, or CT or MRI angiograms. However, clinically asymptomatic vessel thrombosis is sometimes picked up by screening investigations or during routine imaging for other indications. Acute management of thrombosis and thromboembolism comprises a variety of approaches, including simple observation, treatment with unfractionated or low molecular weight heparin, as well as more aggressive interventions such as thrombolytic therapy or catheter-directed revascularization. Long-term follow-up is dependent on the underlying diagnosis. In the majority of infants, stabilization of the patients' general condition and hemodynamics, which allows removal of indwelling catheters, renders long-term anticoagulation superfluous. Nevertheless, in certain types of congenital heart disease or inherited thrombophilia, long-term prophylaxis may be warranted. This review article focuses on pathophysiology, diagnosis, and acute and long-term management of thrombosis in critically ill term and preterm neonates.
\end{abstract}

Keywords: thrombosis, neonate, pathophysiology, diagnosis, therapy

\section{Introduction}

The group of critically ill newborns presents the largest patient population in childhood suffering from thromboembolism. Making decisions regarding therapeutic strategies is a challenge for the intensive care physician as the clinical significance of neonatal thrombosis varies from asymptomatic incidents to life-or limb-threatening events; moreover, appropriate evidence-based treatment algorithms are lacking.

This review article focuses on the pathophysiology, diagnosis and acute, as well as long-term, management of thrombosis in critically ill term and preterm neonates.

\section{Incidence}

A commonly cited paper published in 1995 reported that approximately 2.4/1000 of newborns admitted to neonatal intensive care units experience a symptomatic thromboembolic event (TE) (Schmidt and Andrew 1995). Another study from Germany reported an incidence of 5.1 per 100.000 live births in the period from 1992 to 1994 (Nowak-Gottl et al 1997). However, these numbers are expected to have been on the rise since that time as a result of ever more invasive therapeutic interventions, including an increasing use of central venous catheters (CVC), venous and arterial umbilical catheters (UVC and UAC), as well as other arterial catheters. For example, today $15 \%$ of all babies admitted to neonatal intensive care unit (NICU) 
and $50 \%$ of all preterm neonates with a birth weight of $<1000 \mathrm{~g}$ are instrumented with an umbilical venous catheter (Seguin et al 1994). Unfortunately, together with asphyxia, sepsis, and prematurity, catheterization is among the most important risk factors for thrombosis in the neonatal period (Nowak-Gottl et al 1997; Kuhle et al 2004).

It is very difficult to estimate the true incidence of CVC-associated TE. While clinical studies report incidences from $13 \%$ up to $30 \%$ (Tanke et al 1994; Roy et al 2002), autopsy findings report UVC-related TE in 20\% to $65 \%$ of neonates who have an UVC in place at the time of demise (Tanke et al 1994; Khilnani et al 1991; Schmidt and Zipursky 1984). In a study investigating children who underwent umbilical vein catheterization, clinically silent portal vein thrombosis (PVT) was found in astonishingly many, namely $43 \%$, of neonates studied. Complete or partial revascularization occurred in only $56 \%$ of these patients (Kim et al 2001). Another study investigating children with PVT found that $73 \%$ had a history of UVC placement (Morag et al 2006).

As in venous TE, the incidence of arterial thrombosis is extremely dependent on the method used to diagnose the event. Whereas clinical signs and symptoms occur in $1 \%-3 \%$ of babies with UAC, ultrasound-based studies report an incidence of $14 \%$ to $35 \%$. Ion angiographic investigations, on the other hand, report an incidence of up to $64 \%$ (Cohen et al 1995).

There are only few studies published on the distribution of neonatal thrombosis or TEs. The synopsis of these few reports is that whereas arterial TEs other than neonatal stroke or renal artery thrombosis are usually found at the site of insertion of an arterial catheter or arterial vascular access, venous thromboses are predominantly located in the renal vein, portal vein, and the inferior and superior vena cava (Nowak-Gottl et al 1997). Other frequently affected locations include the femoral and axillary veins and the right atrium.

Neonatal arterial ischemic stroke is most frequently caused by large vessel infarcts involving the carotid circulation in more than 2/3 of cases (Andrew et al 2001). In many of these infants, either perinatal complications or cardiac disease can be identified (Golomb et al 2001).

\section{Pathophysiology}

If we consider the multiple additive factors that contribute to the development of thromboembolism in the critically ill neonate, it becomes obvious why the neonatal period is more prone to symptomatic or asymptomatic TE than all other periods in infancy, childhood and early adulthood.

\section{Developmental hemostasis}

The concept of the hemostatic system in a dynamic development during infancy and childhood was first introduced by Andrew and colleagues in 1988 and 1990 (Andrew et al 1988; Andrew et al 1990). These changes in plasmatic, cellular and vessel wall components of hemostasis are obviously important in diagnosis, treatment and prevention of thrombosis in a critically ill neonate.

Although the hemostatic system of the term and preterm neonate is significantly different from that of children and adults, most authors refer to the hemostatic potential of the "healthy" neonate as being balanced, neither promoting hemorrhage nor thrombosis.

On the pro-coagulant side, especially the vitamin K-dependent coagulation factors (II, VII, IX, and X) and the components of the contact system (FXI, FXII, prekallikreine and high molecular weight kininogen) show significantly reduced plasma activities in neonates compared to children and adults. Although interpretation of values requires caution as corrected gestational age plays a major role, plasma activities of these components can be as low as 30\% in the neonate versus $80 \%$ to $120 \%$ in adults. Adult plasma concentrations are not achieved before the sixth month of life. However, the vitamin K-dependent inhibitors of coagulation, protein $\mathrm{C}$ and protein $\mathrm{S}$, are also reduced in the neonatal period, counterbalancing the reduced clotting potential of neonatal plasma. In fact, both antithrombin and protein $\mathrm{C}$ concentrations are decreased to approximately $30 \%$ of adult values in term and even lower in preterm newborns. Neonatal platelets have been reported to be hypo-reactive; however, this deficiency seems to be balanced by increased von Willebrand factor activity, resulting in overall normal platelet function.

The activity of the fibrinolytic system in the newborn is reduced compared to adults and older children due to both decreased plasma activity of plasminogen and increased plasma levels of plasminogen activator inhibitor (PAI). The latter fact may explain the high rate of TE associated with intravascular devices in newborns; however, to date there is no evidence that the neonatal hemostatic system either protects from or promotes thrombus formation. Of course, additional risk factors, eg, critical illness or congenital thrombophilia, have to be considered separately from the immaturity of neonatal hemostasis. Given the significant differences between the plasma factor concentrations in different age groups, a detailed knowledge on the development of hemostasis is critical for the intensivist in order to adapt pharmacological approaches and interpret results from 
laboratory tests in the neonate with TE (Ignjatovic et al 2006; Ignjatovic et al 2007).

\section{Acquired thrombophilia in critical illness and sepsis}

Critical illness is a well-recognized risk factor for TE in all age groups. Immobilization, rapid changes in intravascular volume and extensive intravascular instrumentation contribute to the enhanced risk of venous and arterial thrombosis in patients in intensive care units. In neonates, this risk is further increased by the fact that indwelling catheters are large compared to the diameter of the vessel, in most cases obstructing approximately $50 \%$ of the lumen. Hence, it is not surprising that almost $90 \%$ of thromboembolic events in neonates are caused by intravascular devices.

Additional risk factors include, but are not limited to, asphyxia, maternal diabetes, poor cardiac output and dehydration. Neonates are born with a high hematocrit and tend to contract their intravascular volume within the first days of life, making them even more prone to thromboembolic events.

Sepsis is a significant cause of morbidity and mortality in neonates and in adults. The annual combined mortality caused by sepsis in all age groups equals the death rate of myocardial infarction (Angus et al 2001). Sepsis alone is responsible for $7 \%$ of all childhood deaths (Watson et al 2003). In the neonatal population, sepsis is particularly devastating, as this disease causes $45 \%$ of late deaths in the NICU (Meadow et al 2002). Neonates with sepsis develop an acquired pro-thrombotic state due to increased consumption of already limited supplies of coagulation inhibitors. Furthermore, plasma activity of PAI-1 is increased in sepsis and levels of protein $\mathrm{C}$ are reduced. The latter fact has been reported to correlate with poor outcomes in adults and neonates. Ongoing consumption of coagulation factors and platelets resulting in microcirculatory thrombosis likely contributes to sepsis-induced multi-organ failure and death. Macro-circulatory thrombotic events are rare in this setting but can occur, especially in babies who have UACs or UVCs in situ.

\section{Congenital thrombophilia}

Whereas congenital thrombophilia was described not to be associated with UVC thrombosis in one report (Turebylu et al 2007), Heller and colleagues published data from the childhood thrombophilia study group indicating an elevated odds ratio for the presence of congenital thrombophilia in neonates with renal, portal, or hepatic venous thrombosis
(Heller et al 2000). A recent study confirms a high prevalence of inherited thrombophilia in neonatal stroke (Suppiej et al 2007). Heller and colleagues recommended that neonates with TE should undergo an extensive screening, including resistance to activated protein C (APC-R), protein C, protein $\mathrm{S}$, and antithrombin activity, concentration of clottable fibrinogen, plasminogen activity, activities of coagulation factors VIIIC and XII, Lp(a), histidine-rich glycoprotein, heparin cofactor II, antiphospholipid antibodies, lupus anticoagulants, as well as fasting homocysteine concentrations. In addition, DNA-based assays (ie, factor V G1691A mutation, factor II G20210A variant and MTHFR C677T genotype) should be performed (Heller and Nowak-Gottl 2003). Whereas DNA-based mutation analysis can be performed at any time point, protein-based assays should not be carried out in the first 6-8 months after the event and oral anticoagulation is recommended to be discontinued at least 14-30 days before plasma samples for thrombophilia diagnosis are drawn (Heller and Nowak-Gottl 2003).

An obvious clinical picture is associated with either homozygous or double heterozygous congenital protein $\mathrm{C}$ deficiency. These infants develop the life-threatening complication of purpura fulminans within the first days of life (Kreuz et al 1998). Notably, patients with prothrombotic mutations and polymorphisms are most likely to develop thrombosis when triggering elements, such as placement of catheters, prolonged immobilization, or surgery, are also present.

\section{Congenital heart disease}

Infants and young children with cyanotic heart disease are at risk for thrombotic events, including shunt thrombosis, coronary artery thrombosis, and thromboembolic arterial stroke (Israels and Michelson 2006). Cyanotic congenital heart diseases (CHD) with secondary polycythemia and a hyperviscous state are associated with a reduction in blood flow and subsequent thrombosis. In CHD, the incidence of venous TE in infants $<6$ months of age is about $50 \%$ and even higher in arterial TE (70\%) (Monagle 2004). The increasing number of surgical procedures carried out in the neonatal period potentiates the risk for TE events. Trans-catheter interventions in newborns, which are mostly performed using venous access from the femoral or umbilical vein, incur the risk for transient or permanent occlusion of the lower body veins. In complex heart disease with the possibility of right-to-left shunt, paradoxical embolism may occur. Prenatally, the Eustachian valve directs the blood from the umbilical vein and the lower body to the foramen ovale and to the left atrium. Postnatally, emboli may take the 
same route to the systemic circulation, thus increasing the risk for a thromboembolic incident when femoral catheters or UVC are used compared to central venous lines from the upper body.

We and others reported a $12 \%$ incidence of aortopulmonary shunt thrombosis in a prospective multicenter observational study ( $\mathrm{Li}$ et al 2007), indicating a hypercoagulable state of cyanotic children after insertion of intravascular artificial prostheses. Furthermore, acquired thrombophilia in cyanotic children has been described in terms of elevated markers as platelet p-selectin, and decreased levels of thrombomodulin and protein $\mathrm{C}$ activity (Kajimoto et al 2007). Patients with cyanotic congenital heart disease usually undergo a preoperative analysis of the well known genetic aberrations associated with increased thrombotic potential, eg, the Factor V Leiden and the MTHFR mutations. In patients with thrombosis after cardiac surgery, in which the classical screening parameters were normal, Ozbek and colleagues recently described other polymorphisms that included a higher frequency of the prothrombotic angiotensin 1 converting enzyme deletion/deletion genotype, and a lower frequency of the antithrombotic alpha fibrinogen Thr/Thr genotype (Ozbek et al 2005).

\section{Diagnosis}

\section{Clinical}

The clinical picture of vascular incidents in neonates is extremely variable and largely dependent on the location and the size of the thrombus or embolus. Presentation may vary from discrete symptoms or asymptomatic events to life or limb threatening acute events. Although in the following sections TE will be categorized as arterial, venous, and CNS events, one needs to keep in mind that several right-to-left (ie, venoarterial) shunts remain patent for a considerable time even in the term neonate. Thus, a paradoxical embolus cannot be ruled out even in the absence of congenital heart disease.

\section{Arterial}

Arterial vascular events, accounting for about half of all $\mathrm{TE}$ in the neonatal population, are almost always directly related to arterial vascular catheterization of central or peripheral arteries. This underscores the need for meticulous clinical observation in children with arterial access in place. Obvious signs include ischemia of limbs or trunk, pale or cold extremities distal to the catheterization site, weak or absent palpability of the pulse (pulse oximeters will also show this) and decreased or immeasurable blood pressure. Signs of necrotizing enterocolitis such as feed intolerance, bilious gastric aspirates, blood-stained stools, and bowel wall pneumatosis in an infant with UAC should raise the suspicion of mesenteric infarction. Diagnostic work-up for renal failure in children with UAC should include a Doppler flow study of the renal arteries to avoid missing out on the diagnosis of renal artery thrombosis. Elevated blood pressure might also hint at decreased renal perfusion.

There are some reports of aortic thrombosis in the neonate mimicking coarctation of the aorta with significant blood pressure differences in upper and lower body blood pressure readings; thus, the simple investigation of upper and lower body blood pressure measurements should be performed in any infant with hypertension diagnosed by measurements obtained from the upper body.

\section{Venous}

Limb swelling, pain, and cyanotic or hyperemic color should raise the suspicion of venous thrombosis. Renal vein thrombosis may present with an abdominal mass and hematuria or proteinuria; hence, these symptoms should ring a bell especially in neonates with risk factors such as prematurity, asphyxia, critical illness, femoral CVL, and male sex (Kuhle et al 2004; Bokenkamp et al 2000).

Signs of impaired liver function, hepatomegaly and splenomegaly should raise the suspicion of PVT; however, only about $10 \%$ of children with PVT develop acute clinical symptoms (Morag et al 2006). Thrombosis of the inferior vena cava can present with signs resembling obstruction of the renal vein (hematuria, retroperitoneal mass); however, these will occur bilaterally when the inferior vena cava is affected. In addition, the lower limbs may be edematous and, if blood flow is substantially impaired, the child may be in respiratory distress and may have high blood pressure.

\section{Pulmonary embolism}

Pulmonary embolism (PE) is a rare event in the neonatal period. Clinical signs of massive ventilation/perfusion mismatch, difficult oxygenation, and signs of right heart failure should raise the suspicion of PE, especially in babies with congenital heart disease. In the few cases reported in the literature, diagnosis of PE was confirmed by lung perfusion scans or angiography (Feldman et al 2005; Gamillscheg et al 1997; Moreno-Cabral and Breitweser 1983).

\section{Central nervous system}

In contrast to the clinical picture in adults, neonatal stroke does not usually present with hemiplegia. The leading clinical symptoms in central nervous system TE in neonates are seizures and lethargy. The diagnosis of ischemic stroke as well as of sinus vein thrombosis can initially be confirmed 
by cerebral ultrasound through the open fontanel followed by cranial magnetic resonance imaging (MRI).

\section{Post-thrombotic syndrome}

TEs that remain clinically asymptomatic in the neonatal period can become evident later in life when children or adolescents present with venous collaterals, post-thrombotic syndrome (featuring edema, purpura, eczematous dermatitis, pruritus, ulceration, and/or cellulitis), reduced limb growth or other late complications such as portal hypertension. Any of these symptoms can be the result of a vascular accident in the neonatal period. See also Reassessment, below, for more detail (Table 1).

\section{Imaging}

\section{Ultrasound}

Imaging by using either echocardiography or abdominal ultrasound is the most commonly applied diagnostic method to confirm clinical suspicion of TE or to screen babies for clinically silent disease. However, despite its advantages as point of care testing (minimal invasiveness and no use of radiation), the overall performance of ultrasound to detect thrombi is poor. One study comparing echocardiographic investigations to venograms reported a sensitivity of $21 \%-43 \%$ and specificities ranging from $76 \%$ to $94 \%$ (Roy et al 2002). This study concluded that venography is required to accurately diagnose UVC related TE in neonates. Since in this study $4 \%$ of all infants investigated were incorrectly diagnosed as having central venous thrombosis, the confirmation with contrast venography might avoid exposing an infant unnecessarily to potentially hazardous treatment.

\section{Contrast venogram}

Still regarded as the gold standard in the diagnosis of neonatal catheter-induced TE, a contrast venogram exposes the infant to radiation and contrast agent. A recent study, using 1.5 to $2 \mathrm{ml}$ of iohexol ${ }^{\mathrm{TM}}$ administered by hand injection over 1 to $2 \mathrm{sec}$ and fluoroscopy times of 5-15 sec, reported a $100 \%$ agreement between two blinded radiologists about presence or absence of thrombi. In one of 14 babies with thrombosis a disagreement between the two examiners was reported in regards to the extension of the thrombus. A portable image intensifier allows contrast venography to be performed on the NICU as long as radiation exposure guidelines are taken into consideration (Roy et al 2002).

\section{MRI angiography}

MRI angiography is recommended for the diagnosis of ischemic neonatal stroke and is an option in neonatal pulmonary embolism. The need for transport limits the practicability in the most critically ill and very preterm neonatal patients.

\section{Laboratory}

Initial laboratory work-up in a neonate in whom thrombosis is suspected should include a full blood count as

Table I Clinical signs and symptoms of thromboembolism in critically ill neonates

\begin{tabular}{|c|c|c|c|c|c|c|}
\hline Early & Extremities & Intestine & Kidney & Aorta & CNS & Lung \\
\hline \multirow[t]{4}{*}{ Arterial } & Extremities pale & Feed intolerance & Blood pressure & Blood pressure & Lethargy & Right heart failure \\
\hline & and/or cold & Gastric aspirates bilious & elevated & higher in arms & Seizures & Oxygen \\
\hline & Pulse weak or absent & Stools bloody & & than in legs & (NO hemiplegia) & saturation low \\
\hline & $\begin{array}{l}\text { Blood pressure } \\
\text { reduced }\end{array}$ & Bowel wall pneumatosis & & & & $\begin{array}{l}\text { Ventilation/perfusion } \\
\text { mismatch }\end{array}$ \\
\hline \multirow[t]{5}{*}{ Venous } & & Portal vein & & Inferior vena cava & & \\
\hline & Swelling & Liver function impaired & Hematuria & Hematuria & Lethargy Seizures & \\
\hline & Pain & Splenomegaly & Proteinuria & Lower limb edema & & \\
\hline & Cyanosis & & Abdominal mass & Both kidneys palpable & & \\
\hline & Hyperemia & & & Respiratory distress & & \\
\hline \multirow[t]{7}{*}{ Late } & & Portal vein & & Inferior vena cava & & \\
\hline & Venous collaterals & Portal hypertension & & & & \\
\hline & Limb growth & Gl hemorrhage & & Leg and abdominal & Neurodevelop- & Right heart \\
\hline & reduced & Hepatic atrophy & Blood pressure & pain Varicose veins & mental delay & hypertrophy \\
\hline & Post-thrombotic & Splenomegaly & abnormalities & Post-thrombotic & Cognitive & \\
\hline & syndrome & & & syndrome & impairment & \\
\hline & & & & & Cerebral palsy & \\
\hline
\end{tabular}

Abbreviation: Gl, gastrointestinal 
well as a coagulation screening with determination of prothrombin time, thrombin time and activated partial thromboplastin time.

\section{D-dimers}

D-dimers, which are elevated as an acute phase reaction in all patients with infection or systemic inflammatory response syndrome (SIRS), are a positive finding in almost all critically ill neonates. Conversely, negative D-dimers are relatively accurate in ruling out thrombosis in most patients, including neonates.

\section{Platelets}

In almost all neonates, platelet numbers decrease after birth. However, a sudden and severe drop in platelet counts should alert the intensivist. Although the differential diagnosis for thrombocytopenia in the neonate is broad, including auto- and allo-antibodies as well as effects of medications and consumption, thrombocytopenia remains one of the most sensitive indicators for micro- (in the setting of sepsis) or macro-circulatory thrombosis.

\section{Prevention}

Obviously, strategies to prevent thrombus formation are of utmost importance in critically ill neonates. Since the majority of TE in neonates is catheter-related, optimal management and careful consideration of the necessity of these devices might give the intensivist a unique chance to improve outcome. Since even small thrombi might act as a nidus for infection, prevention of clot formation is of double benefit for the patient.

\section{Umbilical catheters}

Data suggest that UAC-related thrombosis can be minimized by high umbilical positioning, end-hole and single-lumen construction (Barrington 2000; Barrington 2000). Low-dose heparin infusion is recommended for UACs with some studies suggesting that even low doses $0.25 \mathrm{IU} / \mathrm{ml}$ are effective (Barrington 2000). The incidence of heparin-induced thrombocytopenia (HIT II) in preterm newborns seems to be extremely low, thus promoting the use of unfractionated heparin (UFH) to ensure line patency (Kumar et al 2004).

Time in situ should be minimalized and many neonatologists would see no indications for a UAC in a baby with stable hemodynamics and low to moderate ventilation parameters (Fletcher et al 1994). Although some reports indicate that indwelling UACs in sick infants may not carry an increased risk of thrombosis during the first 5 days of use (Coleman et al 2004), a study published by Boo and colleagues showed that the only significant risk factor associated with the development of abdominal aortic thrombosis following insertion of UAC was longer duration of UAC in situ (for every additional day of UAC in situ, adjusted OR of developing thrombosis was $1.2,95 \% \mathrm{CI}: 1.1,1.3 ; \mathrm{p}=0.002$ ) (Boo et al 1999). When the use of UVC was audited by the study group for complications in perinatal care, it become apparent that UVC were inserted in approximately $50 \%$ of infants with a birth weight of $1000 \mathrm{~g}$ or less (Seguin et al 1994). Heparin was used in the infusate in the majority of the participating centers. As in UACs, the duration of use seems to be of importance in avoiding thrombus formation. In the report of the study group, the UVC was removed after a median of 4.4 days, the Center for Disease Control recommends removal after 14 days at the latest (O'Grady et al 2002). However, one study recently compared long- and short-term use of UACs in premature neonates and did not find an increased risk of thrombosis in the long-term group in which UACs were in situ for up to 28 days (Butler-O'Hara et al 2006). Nevertheless, 9 of the 45 small for gestational age babies in the study developed a thrombus which was detected by echocardiography.

\section{Other central venous catheters}

Whereas percutanously placed peripheral silastic catheters in general have a lower incidence of thrombosis compared to centrally placed catheters, the highest incidence in CVCs is seen in femoral lines. Internal jugular access shows a lower rate of thrombosis compared to subclavian (Male et al 2005). Heparin infusion is utilized in most centers for CVCs in neonates, especially for those placed in the femoral vein.

The effectiveness of heparin infusion in percutanously placed peripheral silastic catheters has been controversial: A 2005 Cochrane review did not recommend the routine use of heparin for silastic catheters (Shah and Shah 2005), whereas a recently published prospective study documented lower catheter occlusion rates but similar incidences of thrombosis in the heparin vs the placebo group (Shah et al 2007). These findings lead to the publication of an update of the 2005 Cochrane review in 2008, in which the use of heparin at a dose of $0.5 \mathrm{IU} / \mathrm{kg} / \mathrm{h}$ is recommended to maintain line patency, although this regimen does not reduce the risk of catheter-related thrombosis (Shah and Shah 2008).

Heparin-coated catheters might become an interesting option in the future to prevent both catheter occlusion as well as catheter-related thrombosis. However, a recent 
Cochrane review could not yet confirm a clear advantage of heparin-coated catheters in children (Shah and Shah 2007) and studies in neonates have not yet been undertaken.

\section{Management}

Acute

When to treat TE in critically ill neonates is a challenging question for everybody involved in the care of these infants. Since withholding anticoagulation is an equally active decision as commencing treatment, the individual risk/benefit ratio has to be carefully considered.

Potential benefits of antithrombotic therapy include complete or partial re-canalization of the obstructed vessel with consequently decreased tissue hypoperfusion in the affected area; limb saving in the scenario of acute arterial occlusion; avoidance of detrimental long term morbidity from conditions such as portal hypertension, hepatic post thrombotic syndrome, renal hypertension, impaired renal function, renal shrinkage, discrepancies in limb growth, claudication, and paraplegia.

The major potential risk of antithrombotic therapy, especially in neonates, is a hemorrhagic event that is potentially massive and life threatening, in particular intracranial hemorrhage. Since low molecular weight heparins show a particularly favorable risk/benefit ratio, the decision not to treat TE will most likely be restricted to those infants with an exceptionally high risk of hemorrhage, eg, very immature preterm neonates within the first days of life or established intraventricular hemorrhage (IVH). Other relative contraindications for anticoagulant therapy include thrombocytopenia with platelet counts of less than $50 \mathrm{G} / \mathrm{L}$ and history of high grade IVH. In most other patients, heparin therapy might be considered appropriate for clinically symptomatic TE.

The appropriateness of thrombolytic therapy will always depend on the individual patient. However, since the risk of hemorrhage, especially in systemic allied thrombolytic therapy remains unclear, most would restrict this therapeutic approach to life or limb threatening vessel obstructions.

Guidelines on treatment of clinically asymptomatic clots (detected for example during routine imaging in critically ill neonates) are not published and individual decision-making remains necessary. A majority of clinicians might decide to treat clots which obstruct more than $50 \%$ of the lumen of a major vessel (including the portal venous system) with low molecular weight heparin (LMWH) in the absence of contraindications. This approach takes both the potential long-term consequences of even asymptomatic TE in major vessels (see Follow-up, below) as well as the potential danger that the thrombus size may increase into account.

\section{Medical}

\section{Unfractionated heparin}

Unfractionated heparin has been the standard medication for prophylaxis and treatment of TE for many years. Due to unfavorable pharmacokinetics and pharmacodynamics and the imminent danger of HIT, UFH was almost completely replaced by low molecular weight heparins in adult medicine with the exception of anticoagulation during extracorporal circulation. In neonatal medicine, however, UFH are still widely used to prevent and treat TE. Despite the lack of studies on LMWH in the neonatal population, the low incidence of HIT II (Kumar et al 2004), and the availability of protamine for potential reversal of UFH action, support its use in preterm and term neonates. Dosages of $>25 \mathrm{IU} / \mathrm{kg} / \mathrm{h}$ are usually required to achieve a targeted prolongation of the aPTT to $70 \mathrm{sec}$ and above.

\section{Low molecular weight heparin}

Low molecular weight heparin has widely replaced UFH in prophylaxis and treatment of acute TE in adult patients due to superior efficacy, safety and pharmacokinetics. In neonatal patients, the data available are still limited. However, LMWH is increasingly used in NICU in the post-acute treatment of venous and arterial TE as well as in prevention of thrombosis in high-risk patients. Subcutaneous enoxaparin seems to be the most frequently used substance in term and preterm neonates. Data published on the use of enoxaparin in 12 preterm and 4 term infants found no serious adverse events and a resolution of TE in 71\% of the patients (Malowany et al 2007).

Anti-Xa level is usually targeted at $0.4-0.6 \mathrm{IU} / \mathrm{ml}$ in prophylaxis and at $0.6-0.8 \mathrm{IU} / \mathrm{ml}$ in treatment scenarios. Preterm infants require longer to achieve an anti-Xa level within target range compared to term infants (6 vs 2 days) and were found to require higher doses of enoxaparin $(2.1 \mathrm{mg} / \mathrm{kg} / 12 \mathrm{~h}$ vs $1.7 \mathrm{mg} / \mathrm{kg} / 12 \mathrm{~h}$ ) (Streif et al 2003). Recent evidence also suggests a starting dose of $1.7 \mathrm{mg} / \mathrm{kg} / 12 \mathrm{~h}$ in term and $2 \mathrm{mg} / \mathrm{kg} / 12 \mathrm{~h}$ in preterm neonates (Malowany et al 2008). Efficacy was reported to be 59\%-100\%. Cessation of anticoagulation can be achieved by terminating the medication $12-24 \mathrm{~h}$ prior to planned surgical procedures. In contrast to common belief, protamine is able to antagonize anti-Xa activity of LMWH in part and anti-IIa activity of LMWH completely (Crowther et al 2002). In a recent case report, an overdose of $40 \mathrm{mg}$ of enoxaparin in a neonate was successfully antagonized with $35 \mathrm{mg}$ of protamine given in the preceding 4.5-7.5 h (Wiernikowski et al 2007). 


\section{Streptokinase}

Streptokinase is infrequently administered in the neonatal period due to decreased fibrin specificity and high antigenicity compared to rtPA. In the few patients described in the literature, doses of 1000-2000 IU/ $\mathrm{kg} / \mathrm{h}$ have been used (Nowak-Gottl et al 1997; Hausler et al 2001).

\section{rtPA (recombinant tissue plasminogen activator)}

If thrombolytic therapy is utilized, rtPA is mostly favored due to high fibrin specificity with poor activation of free plasmin, lack of antigenicity and short half life (4 min in plasma, $45 \mathrm{~min}$ for thrombolytic effects). Dosing varies between different reports, ranging from 0.3 to $9 \mathrm{mg} / \mathrm{kg} / 24 \mathrm{~h}$. Plasminogen levels are low in neonates compared to adults, pointing towards a possible requirement for higher doses of rtPA. Administration of fresh frozen plasma (FFP) prior to utilization of thrombolytics may increase success rates by providing sufficient plasminogen levels. Frequent monitoring of fibrinogen levels and FFP administration if fibrinogen levels fall below $100 \mathrm{mg} / \mathrm{dl}$ are required. For safety and efficacy of systemic thrombolysis in neonates see below.

\section{Urokinase}

As with streptokinase, the decreased fibrin specificity and the antigenicity of urokinase compared to rtPA limit its use in neonates. In the small number of neonatal patients which are described in the literature, doses of $1000 \mathrm{up}$ to $10.000 \mathrm{IU} / \mathrm{kg} / \mathrm{h}$ have been reported, although the majority of patients received $2000 \mathrm{IU} / \mathrm{kg} / \mathrm{h}$ or less (Nowak-Gottl et al 1997; Hausler et al 2001; Duncan et al 1991; Griffin and Casta 1988; Distefano et al 2000; Kobayashi et al 2006).

Some studies, which include small numbers of term neonates, investigated urokinase as treatment or prevention of catheter occlusion and/or catheter infection with some success in older children and adults (Dillon et al 2004; Svoboda et al 2004). However, this treatment is not sufficiently evaluated in the neonatal population to be recommended.

\section{Duration of treatment}

The administration of fibrinolytics such as rtPA, streptokinase, and urokinase is usually rather short in duration. These agents are stopped either after a few days or when revascularization has been achieved (as determined clinically or via imaging). In contrast, LMWH is increasingly used in therapeutic concentrations for prolonged therapies of 6 weeks or even up to 3 months, since late revascularization occurs in adults (Agnelli and Becattini 2008; Hull et al 2006) and in children and neonates, as the few studies performed in these patient groups have shown (Nowak-Gottl et al 2008).

\section{Interventional}

\section{Catheter-directed thrombolysis}

Catheter-directed thrombolysis (CDT) has been reported in 13 patients in the neonatal period (Anderson et al 1991; Aspesberro et al 1999; Dillon et al 1993; Gerling et al 2002; Khan et al 2008; Klinge et al 1998; Pritchard et al 1985; Rehan et al 1994; Ries et al 2002; Rogers et al 1989; Ryan and Andrew 1992; Wang et al 2003). In these reports, rtPA (dosage $0.01-0.1-0.5 \mathrm{mg} / \mathrm{kg} / \mathrm{h}$ [min-median-max]) was used in 8 patients, while 4 patients received streptokinase and one was treated with urokinase. In contrast to systemic thrombolytic therapy, efficacy of CDT seems to be superior ( $82 \%$ vs $62 \%$ of complete clot lysis, $16 \%$ vs $8 \%$ partial clot lysis, $8 \%$ vs $21 \%$ failure). The safety profile also favors CDT with a rate of one intraventricular hemorrhage (IVH) in 13 patients with CDT compared to $2 \mathrm{IVH}$, one intra abdominal bleed, one kidney rupture, one mucosal bleed and one puncture site bleed in 38 neonates with systemic thrombolysis (Khan et al 2008).

\section{Other catheter interventions}

Mechanical thrombectomy has been reported in a neonate with pulmonary embolism after surgery for congenital heart disease (Moreno-Cabral and Breitweser 1983). Availability of small catheters (4F) allow nonwall-contact procedures, breaking the thrombus by circulating fluids and thrombus removal by suctioning and aspiration of the thrombotic material (Angiojet, Possis, Hydrolizer, Cordis). Successful hydrodynamic (Angiojet) catheter-based thrombectomy has been described in neonates with postoperative pulmonary embolism (Feldman et al 2005; Kobayashi et al 2006). Possible side effects such as hemolysis, blood loss and bradycardia may be more pronounced in neonates. The use of the classical Fogarty catheter may be useful in arterial limb ischemia in neonates $>1500 \mathrm{~g}$. Wall contact procedures such as clot maceration brushes have not been used in the neonatal period and carry in general the risk of late post-thrombotic syndrome, when applied in venous thrombosis due to rupture of venous valves (Figure 1).

\section{Surgical}

Surgical clot removal and vessel reconstruction may become necessary in the rare event of life or limb threatening arterial thromboembolism and in the even rarer event of massive venous thrombosis. A treatment protocol including surgical intervention for infants with brachial artery thrombosis has been published by Coombs and colleagues (Coombs et al 2006). However, due to the high rate of re-occlusion of the 


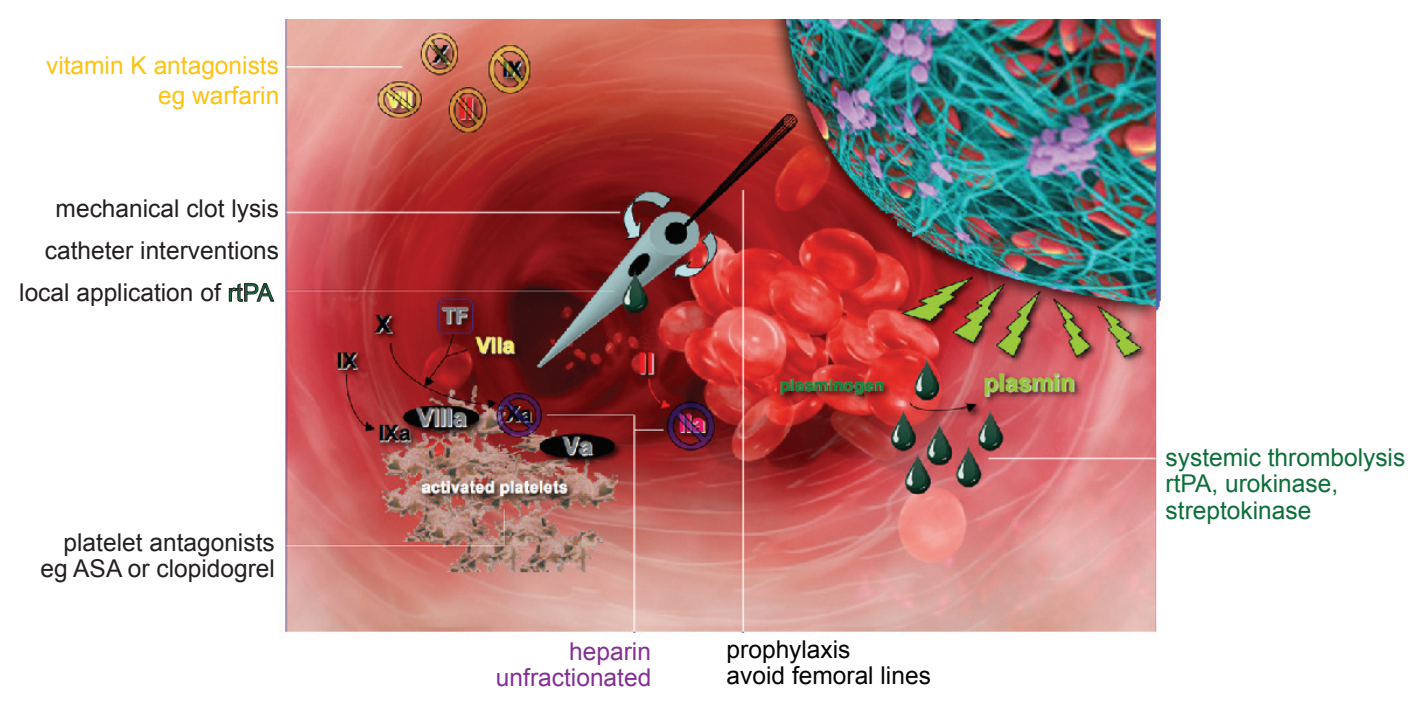

Figure I Therapeutic options and mechanisms of action.

affected vessel, embolectomy is avoided if at all possible (Monagle et al 2004).

Successful surgical embolectomy has been described in neonates with life threatening pulmonary embolism (Gamillscheg et al 1997; Moreno-Cabral and Breitweser 1983).

\section{Follow-up}

\section{Long-term anticoagulation}

In the absence of solid data the need for long-term anticoagulation in neonates with symptomatic TE is debatable. Most clinicians would agree not to treat catheter-induced thrombosis in a patient without congenital thrombophilia with long-term anticoagulation. However, in patients with single prothrombotic traits, anticoagulation for 3 to 12 months after the onset of the event is recommended (Heller and Nowak-Gottl 2003). Although data published by Kurnik and colleagues give evidence that symptomatic recurrent thromboembolism is not common in children with neonatal arterial ischemic stroke(Kurnik et al 2003), anticoagulation with LMWH or low dose aspirin in children with arterial vascular occlusion or ischemic stroke is recommended by some (Heller and Nowak-Gottl 2003; Strater et al 2001).

\section{Warfarin}

The use of warfarin in the neonatal period is problematic. Many formula fed infants receive large amounts of vitamin K in the formula and thus are almost resistant to warfarin. Dosing in small children is difficult since liquid formulations are not commercially available and customized suspension of tablets does not provide sufficient accuracy in dosing. If warfarin is used beyond the neonatal period, training of parents in point of care coagulation test devices in patient self control programs can be of great help in ensuring INR levels within the desired target range.

\section{Aspirin}

Platelet function is reduced in neonates in comparison to adult and older children. Anti-platelet agents such as aspirin or the newer agent clopidogrel are traditionally used in occlusion prophylaxis in patients with congenital heart disease and shunt-dependent pulmonary or systemic circulation. In contrast to the use of aspirin as prophylaxis in patients with a history of TE, there is no evidence that anti-platelet therapy is effective as treatment in acute venous or arterial neonatal TE. Neonates with cyanotic congenital heart disease palliated with a shunt who present with abnormal laboratory findings suggestive of an increased risk for developing thrombosis should be treated with platelet aggregation inhibitors.

\section{Clopidogrel}

Clopidogrel (Plavix), a thienopyridine derivative, selectively inhibits the binding of ADP to its platelet receptor and the subsequent ADP-mediated activation of the glycoprotein IIb/IIIa complex, thereby inhibiting platelet aggregation and reducing the formation of arterial and venous thrombi (Coukell and Markham 1997). Recently a prospective, multicenter, randomized, double-blind, placebo-controlled, dose-ranging study showed a dosage of $0.2 \mathrm{mg} / \mathrm{kg}$ once daily to be effective in neonates and infants with CHD in terms of achieving a mean of $30 \%$ to $50 \%$ inhibition of ADP-induced platelet aggregation ( $\mathrm{Li}$ et al 2008). Administration of higher doses of clopidogrel, extrapolated from adult dosing carries 
the risk of bleeding in high risk patients especially when used in conjunction of Aspirin (Soman et al 2006). Side effects of clopidogrel seem to be lower than in the other thrombocyte aggregation blocking agents. An upcoming study evaluating the efficacy in preventing TE will elucidate the risk and benefit of this drug in neonates and infants.

\section{Re-assessment}

Portal hypertension (PHTN), which is the major cause of gastrointestinal (GI) hemorrhage in children, is the one of the most severe long-term consequences of UVC associated portal vein obstruction. PHTN is diagnosed at a mean of 5.7 years after the acute event (Alvarez et al 1983). Children with portal obstruction have a history of UVC placement in the neonatal period in $70 \%$ of the cases (Morag et al 2006). It might be worthwhile to investigate children with UVC history for signs of PHTN if left lobe hepatic atrophy, splenomegaly, thrombocytopenia or gastrointestinal hemorrhage is noticed.

The occurrence of post-thrombotic syndrome is increasingly reported in follow-up studies in preterm neonates. In a study published by Hausler and colleagues following up on 40 infants and children with IVC thrombosis complete restitution was found in only four cases. Persisting iliac and/or caval venous obstruction occurred in 17 patients, including six with initial thrombolysis. Varicose veins were found in 12, and postthrombotic syndrome in seven of these cases. According to Kaplan-Meier analysis, 30\% of patients with persisting venous disease will develop post-thrombotic syndrome within 10 years of the thrombotic event (Hausler et al 2001). This underlines the importance of achieving the best possible vascular status in the initial treatment attempt, since post-thrombotic syndrome can be prevented by successful thrombolysis in children (Goldenberg et al 2007). Consequently, the risk of treatment in the initial event must be carefully judged against the risk of missing out on treatment.

Long-term complications of arterial thrombosis include hypertension, impaired renal function, discrepancies in limb growth, claudication and paraplegia. Patients with a history of arterial thrombosis therefore need a regular follow-up.

\section{Conclusion}

Critically ill neonates are at major risk of venous and arterial thromboembolic events. Since indwelling catheters are the main contributing factor, a significant proportion of these events might be preventable by critically re-evaluating especially the indication for umbilical artery and venous catheter on a daily basis and using heparin prophylaxis in those babies where catheterization of the umbilical vessels cannot be discontinued.

While therapy of established thrombus formation with unfractionated or low molecular weight heparin needs to be weighed carefully against the "lost chance" of not applying therapy, thrombolysis or mechanical clot removal should be restricted to the rare scenarios of acute life threatening vessel occlusion.

All neonates with a history of thromboembolic events warrant a follow-up with an emphasis on the development of post-thrombotic syndrome, portal hypertension and an investigation for congenital thrombophilic risk factors. A detailed knowledge of developmental hemostasis is necessary to guide therapy and monitoring in neonates with thrombotic events.

\section{Disclosure}

The authors report no conflicts of interest in this work.

\section{References}

Agnelli and Becattini C. 2008. Treatment of DVT: how long is enough and how do you predict recurrence. J Thromb Thrombolysis, 25:37-44.

Alvarez F, Bernard O, Brunelle F, et al. 1983. Portal obstruction in children. I. Clinical investigation and hemorrhage risk. J Pediatr, 103:696-702.

Anderson BJ, Keeley SR, Johnson ND, et al. 1991. Caval thrombolysis in neonates using low doses of recombinant human tissue-type plasminogen activator. Anaesth Intensive Care, 19:22-7.

Andrew M, Paes B, Milner R, et al. 1988. Development of the human coagulation system in the healthy premature infant. Blood, 72:1651-7.

Andrew M, Paes B, Johnston M, et al. 1990. Development of the hemostatic system in the neonate and young infant. Am J Pediatr Hematol Oncol, 12:95-104.

Andrew ME, Monagle P, deVeber G, et al. 2001. Thromboembolic disease and antithrombotic therapy in newborns. Hematology Am Soc Hematol Educ Program, 358-74.

Angus DC, Linde-Zwirble WT, Lidicker J, et al. 2001. Epidemiology of severe sepsis in the United States: analysis of incidence, outcome, and associated costs of care. Crit Care Med, 29:1303-10.

Aspesberro F, Beghetti M, Oberhansli I, et al. 1999. Local low-dose urokinase treatment of acquired intracardiac thrombi in preterm infants. Eur J Pediatr, 158:698-701.

Barrington KJ. 2000. Umbilical artery catheters in the newborn: effects of position of the catheter tip. Cochrane Database Syst Rev, 2: CD000505.

Barrington KJ. 2000. Umbilical artery catheters in the newborn: effects of heparin. Cochrane Database Syst Rev, 2:CD000507.

Barrington KJ. 2000. Umbilical artery catheters in the newborn: effects of catheter design (end vs side hole). Cochrane Database Syst Rev, 2:CD000508.

Bokenkamp A, von KR, Nowak-Gottl U, et al. 2000. Neonatal renal venous thrombosis in Germany between 1992 and 1994: epidemiology, treatment and outcome. Eur J Pediatr, 159(1-2):44-8.

Boo NY, Wong NC, Zulkifli SS, et al. 1999. Risk factors associated with umbilical vascular catheter-associated thrombosis in newborn infants. $J$ Paediatr Child Health, 35:460-5.

Butler-O'Hara M, Buzzard CJ, Reubens L, et al. 2006. A randomized trial comparing long-term and short-term use of umbilical venous catheters in premature infants with birth weights of less than 1251 grams. Pediatrics, 118:e25-e35. 
Cohen RS, Ramachandran P, Kim EH, et al. 1995. Retrospective analysis of risks associated with an umbilical artery catheter system for continuous monitoring of arterial oxygen tension. J Perinatol, 15:195-8.

Coleman MM, Spear ML, Finkelstein M, et al. 2004. Short-term use of umbilical artery catheters may not be associated with increased risk for thrombosis. Pediatrics, 113:770-4.

Coombs CJ, Richardson PW, Dowling GJ, et al. 2006. Brachial artery thrombosis in infants: an algorithm for limb salvage. Plast Reconstr Surg. 117:1481-8.

Coukell AJ and Markham A. 1997. Clopidogrel. Drugs, 54:745-50.

Crowther MA, Berry LR, Monagle PT, et al. 2002. Mechanisms responsible for the failure of protamine to inactivate low-molecular-weight heparin. Br J Haematol, 116:178-86.

Dillon PW, Fox PS, Berg CJ, et al. 1993. Recombinant tissue plasminogen activator for neonatal and pediatric vascular thrombolytic therapy. $J$ Pediatr Surg, 28:1264-8.

Dillon PW, Jones GR, Bagnall-Reeb HA, et al. 2004. Prophylactic urokinase in the management of long-term venous access devices in children: a Children's Oncology Group study. J Clin Oncol, $22: 2718-23$

Distefano G, Rodono A, Cilauro S, et al. 2000. Fibrinolytic treatment of portal vein thrombosis after umbilical catheterization using systemic urokinase. Pediatr Int, 42:82-4.

Duncan BW, Adzick NS, Longaker MT, et al. 1991. In utero arterial embolism from renal vein thrombosis with successful postnatal thrombolytic therapy. J Pediatr Surg, 26:741-3.

Feldman JP, Feinstein JA, Lamberti JJ, et al. 2005. Angiojet catheter-based thrombectomy in a neonate with postoperative pulmonary embolism. Catheter Cardiovasc Interv, 66:442-5.

Fletcher MA, Brown DR, Landers S, et al. 1994. Umbilical arterial catheter use: report of an audit conducted by the Study Group for Complications of Perinatal Care. Am J Perinatol, 11:94-9.

Gamillscheg A, Nurnberg JH, exi-Meskishvili V, et al. 1997. Surgical emergency embolectomy for the treatment of fulminant pulmonary embolism in a preterm infant. J Pediatr Surg, 32:1516-8.

Gerling S, Klinge J, Singer H, et al. 2002. Successful treatment of superior vena cava thrombosis in a neonate. Eur J Pediatr, 161:466-7.

Goldenberg NA, Durham JD, Knapp-Clevenger R, et al. 2007. A thrombolytic regimen for high-risk deep venous thrombosis may substantially reduce the risk of postthrombotic syndrome in children. Blood, 110:45-53.

Golomb MR, MacGregor DL, Domi T, et al. 2001. Presumed pre- or perinatal arterial ischemic stroke: risk factors and outcomes. Ann Neurol, 50:163-8.

Griffin MP and Casta A. 1988. Successful urokinase therapy for superior vena cava syndrome in a premature infant. Am J Dis Child, $142: 1267-8$.

Hausler M, Hubner D, Delhaas T, et al. 2001. Long term complications of inferior vena cava thrombosis. Arch Dis Child, 85:228-33.

Hausler M, Hubner D, Hornchen H, et al. 2001. Successful thrombolysis of inferior vena cava thrombolysis in a preterm neonate. Clin Pediatr (Phila), 40:105-8.

Heller C, Schobess R, Kurnik K, et al. 2000. Abdominal venous thrombosis in neonates and infants: role of prothrombotic risk factors - a multicentre case-control study. For the Childhood Thrombophilia Study Group. $\mathrm{Br}$ J Haematol, 111:534-9.

Heller C and Nowak-Gottl U. 2003. Maternal thrombophilia and neonatal thrombosis. Best Pract Res Clin Haematol, 16:333-45.

Hull RD, Pineo GF, Brant RF, et al. 2006. Long-term low-molecular-weight heparin versus usual care in proximal-vein thrombosis patients with cancer. Am J Med, 119:1062-72.

Ignjatovic V, Summerhayes R, Than J, et al. 2006. Therapeutic range for unfractionated heparin therapy: age-related differences in response in children. J Thromb Haemost, 4:2280-2.

IIgnjatovic V, Summerhayes R, Gan A, et al. 2007. Monitoring unfractionated Heparin (UFH) therapy: which anti-factor Xa assay is appropriate? Thromb Res, 120:347-51.
Israels SJ and Michelson AD. 2006. Antiplatelet therapy in children. Thromb Res, 118:75-83.

Kajimoto H, Nakazawa M, Murasaki K, et al. 2007. Increased thrombogenesity in patients with cyanotic congenital heart disease. Circ J, 2007;71:948-53.

Khan JU, Takemoto CM, Casella JF, et al. 2008. Catheter-directed thrombolysis of inferior vena cava thrombosis in a 13-day-old neonate and review of literature. Cardiovasc Intervent Radiol, 31(Suppl 2): S153-60.

Khilnani P, Goldstein B, Todres ID, et al. 1991. Double lumen umbilical venous catheters in critically ill neonates: a randomized prospective study. Crit Care Med, 19:1348-51.

Kim JH, Lee YS, Kim SH, et al. 2001. Does umbilical vein catheterization lead to portal venous thrombosis? Prospective US evaluation in 100 neonates. Radiology, 219:645-50.

Klinge J, Scharf J, Rupprecht T, et al. 1998. Selective thrombolysis in a newborn with bilateral renal venous and cerebral thrombosis and heterozygous APC resistance. Nephrol Dial Transplant, 13:3205-7.

Kobayashi T, Kobayashi T, Mayuzumi H, et al. 2006. Percutaneous hydrodynamic thrombectomy for congenital deep vein thrombosis in a neonate Pediatr Cardiol, 27:170-4.

Kreuz W, Veldman A, Escuriola-Ettingshausen C, et al. 1998. Protein-C concentrate for meningococcal purpura fulminans. Lancet, 351:986-7.

Kuhle S, Massicotte P, Chan A, et al. 2004. A case series of 72 neonates with renal vein thrombosis. Data from the 1-800-NO-CLOTS Registry. Thromb Haemost, 92:729-33.

Kumar P, Hoppensteadt DA, Prechel MM, et al. 2004. Prevalance of heparin-dependent platelet-activating antibodies in preterm newborns after exposure to unfractionated heparin. Clin Appl Thromb Hemost, 10:335-9.

Kurnik K, Kosch A, Strater R, et al. 2003. Recurrent thromboembolism in infants and children suffering from symptomatic neonatal arterial stroke: a prospective follow-up study. Stroke, 34:2887-92.

Li JS, Yow E, Berezny KY, et al. 2007. Clinical outcomes of palliative surgery including a systemic-to-pulmonary artery shunt in infants with cyanotic congenital heart disease: does aspirin make a difference? Circulation, 116:293-7.

Li JS, Yow E, Berezny KY, et al. 2008. Dosing of clopidogrel for platelet inhibition in infants and young children: primary results of the Platelet Inhibition in Children On cLOpidogrel (PICOLO) trial. Circulation. 117:553-9.

Male C, Julian JA, Massicotte P, et al. 2005. Significant association with location of central venous line placement and risk of venous thrombosis in children. Thromb Haemost, 94:516-21.

Malowany JI, Knoppert DC, Chan AK, et al. 2007. Enoxaparin use in the neonatal intensive care unit: experience over 8 years. Pharmacotherapy, $27: 1263-71$

Malowany JI, Monagle P, Knoppert DC, et al. 2008. Enoxaparin for neonatal thrombosis: A call for a higher dose for neonates. Thromb Res, Epub. Jan 17.

Meadow W, Frain L, Ren Y, et al. 2002. Serial assessment of mortality in the neonatal intensive care unit by algorithm and intuition: certainty, uncertainty, and informed consent. Pediatrics, 109:878-86.

Monagle P, Chan A, Massicotte P, et al. 2004. Antithrombotic therapy in children: the Seventh ACCP Conference on Antithrombotic and Thrombolytic Therapy. Chest, $126(3 \mathrm{Suppl}): 645 \mathrm{~S}-87 \mathrm{~S}$.

Monagle P. 2004. Anticoagulation in the young. Heart, 90:808-12.

Morag I, Epelman M, Daneman A et al. 2006. Portal vein thrombosis in the neonate: risk factors, course, and outcome. J Pediatr, 148:735-9.

Moreno-Cabral RJ, Breitweser JA, et al. 1983. Pulmonary embolectomy in the neonate. Chest, 84:502-4.

Nowak-Gottl U, von KR, Gobel U, et al. 1997. Neonatal symptomatic thromboembolism in Germany: two year survey. Arch Dis Child Fetal Neonatal Ed, 76:F163-7.

Nowak-Gottl U, Bidlingmaier C, Krumpel A, et al. 2008. Pharmacokinetics, efficacy, and safety of LMWHs in venous thrombosis and stroke in neonates, infants and children. Br J Pharmacol, 153:1120-7. 
O'Grady NP, Alexander M, Dellinger EP, et al. 2002. Guidelines for the prevention of intravascular catheter-related infections. Centers for Disease Control and Prevention. MMWR Recomm Rep, 51(RR-10):1-29.

Ozbek N, Atac FB, Yildirim SV, et al. 2005. Analysis of prothrombotic mutations and polymorphisms in children who developed thrombosis in the perioperative period of congenital cardiac surgery. Cardiol Young, 15:19-25.

Pritchard SL, Culham JA, Rogers PC, et al. 1985. Low-dose fibrinolytic therapy in infants. $J$ Pediatr, 106:594-8.

Rehan VK, Cronin CM, Bowman JM, et al. 1994. Neonatal portal vein thrombosis successfully treated by regional streptokinase infusion. Eur J Pediatr, 153:456-9.

Ries M, Zenker M, Girisch M, et al. 2002. Percutaneous endovascular catheter aspiration thrombectomy of severe superior vena cava syndrome. Arch Dis Child Fetal Neonatal Ed, 87:F64-6.

Rogers PC, Silva MP, Carter JE, et al. 1989. Renal vein thrombosis and response to therapy in a newborn due to protein C deficiency. Eur $J$ Pediatr, 149:124-5.

Roy M, Turner-Gomes S, Gill G, et al. 2002. Accuracy of Doppler echocardiography for the diagnosis of thrombosis associated with umbilical venous catheters. J Pediatr, 140:131-4.

Ryan CA and Andrew M. 1992. Failure of thrombolytic therapy in four children with extensive thromboses. Am J Dis Child, 146:187-93.

Schmidt B and Zipursky A. 1984. Thrombotic disease in newborn infants. Clin Perinatol, 11:461-88.

Schmidt B and Andrew M. 1995. Neonatal thrombosis: report of a prospective Canadian and international registry. Pediatrics, 96(5 Pt 1):939-43.

Seguin J, Fletcher MA, Landers S, et al. 1994. Umbilical venous catheterizations: audit by the Study Group for Complications of Perinatal Care. Am J Perinatol, 11:67-70.

Shah P and Shah V. 2005. Continuous heparin infusion to prevent thrombosis and catheter occlusion in neonates with peripherally placed percutaneous central venous catheters. Cochrane Database Syst Rev, 3:CD002772.

Shah PS, Kalyn A, Satodia P et al. 2007. A randomized, controlled trial of heparin versus placebo infusion to prolong the usability of peripherally placed percutaneous central venous catheters (PCVCs) in neonates: the HIP (Heparin Infusion for PCVC) study. Pediatrics, 119:284-e291.
Shah PS and Shah N. 2007. Heparin-bonded catheters for prolonging the patency of central venous catheters in children. Cochrane Database Syst Rev, 4:CD005983

Shah PS and Shah VS. 2008. Continuous heparin infusion to prevent thrombosis and catheter occlusion in neonates with peripherally placed percutaneous central venous catheters. Cochrane Database Syst Rev, 2:CD002772.

Soman T, Rafay MF, Hune S, et al. 2006. The risks and safety of clopidogrel in pediatric arterial ischemic stroke. Stroke, 37:1120-2.

Strater R, Kurnik K, Heller C, et al. 2001. Aspirin versus low-dose low-molecular-weight heparin: antithrombotic therapy in pediatric ischemic stroke patients: a prospective follow-up study. Stroke, 32:2554-8.

Streif W, Goebel G, Chan AK, et al. 2003. Use of low molecular mass heparin (enoxaparin) in newborn infants: a prospective cohort study of 62 patients. Arch Dis Child Fetal Neonatal Ed, 88:F365-70.

Suppiej A, Franzoi M, Gentilomo C, et al. 2007. High prevalence of inherited thrombophilia in 'presumed peri-neonatal' ischemic stroke. Eur J Haematol, 80:71-5.

Svoboda P, Barton RP, Barbarash OL, et al. 2004. Recombinant urokinase is safe and effective in restoring patency to occluded central venous access devices: a multiple-center, international trial. Crit Care Med, 32:1990-6.

Tanke RB, van Megen R, Daniels O, et al. 1994. Thrombus detection on central venous catheters in the neonatal intensive care unit. Angiology, 45:477-80.

Turebylu R, Salis R, Erbe R, et al. 2007. Genetic prothrombotic mutations are common in neonates but are not associated with umbilical catheter-associated thrombosis. J Perinatol, 27:490-5.

Wang M, Hays T, Balasa V, et al. 2003. Low-dose tissue plasminogen activator thrombolysis in children. J Pediatr Hematol Oncol, 25:379-86.

Watson RS, Carcillo JA, Linde-Zwirble WT, et al. 2003. The epidemiology of severe sepsis in children in the United States. Am J Respir Crit Care Med, 167:695-701.

Wiernikowski JT, Chan A, Lo G, et al. 2007. Reversal of anti-thrombin activity using protamine sulfate. Experience in a neonate with a 10-fold overdose of enoxaparin. Thromb Res, 120:303-5. 